\title{
Propagation and dissipation of Alfvén waves in stellar atmospheres permeated by isothermal winds ${ }^{\star}$
}

\author{
A. Verdini ${ }^{1}$, M. Velli ${ }^{1}$, and S. Oughton ${ }^{2}$ \\ 1 Dipartimento di Astronomia e Scienza dello Spazio, Firenze, Italy \\ 2 Department of Mathematics, University of Waikato, Private Bag 3105, Hamilton, New Zeland
}

Received 24 January 2005 / Accepted 21 June 2005

\section{ABSTRACT}

We investigate the nonlinear evolution of Alfvén waves in a radially stratified isothermal atmosphere with wind, from the atmospheric base out to the Alfvénic point. Nonlinear interactions, triggered by wave reflection due to the atmospheric gradients, are assumed to occur mainly in directions perpendicular to the mean radial magnetic field. The nonlinear coupling between waves propagating in opposite directions is modeled by a phenomenological term, containing an integral turbulent length scale, which acts as a dissipative coefficient for waves of a given frequency. Although the wind acceleration profile is not determined self-consistently one may estimate the dissipation rate inside the layer and follow the evolution of an initial frequency spectrum. Reflection of low frequency waves drives dissipation across the whole spectrum, and steeper gradients, i.e. lower coronal temperatures, enhance the dissipation rate. Moreover, when reasonable wave amplitudes are considered, waves of all frequencies damp at the same rate and the spectrum is not modified substantially during propagation. Therefore the sub-Alfvénic coronal layer acts differently when waves interact nonlinearly, no longer behaving as a frequency dependent filter once reflection-generated nonlinear interactions are included, at least within the classes of models discussed here.

Key words. magnetohydrodynamics (MHD) - turbulence - waves

\section{Introduction}

A promising mechanism for heating the open solar corona and fast solar wind which has been proposed over recent years (Velli et al. 1989; Matthaeus et al. 1999) is the development of MHD turbulence driven by the reflection of Alfvén waves. The presence of MHD waves inside the solar corona has been proved indirectly by measurements involving Faraday rotation at distances of $\approx 8 R_{\odot}$ from the sun's surface (Hollweg et al. 1982), while much farther away "in situ" measurement of magnetic and velocity field fluctuations from Helios and Ulysses have revealed a broad developed spectrum for frequencies ranging from $10^{-4} \mathrm{~Hz}$ to $10^{-2} \mathrm{~Hz}$ (for the fast component of the solar wind). Typically, a strong correlation between magnetic field and velocity fluctuations in this distance range persists (Mangeney et al. 1991; Smith et al. 1995). At intermediate distances (from 10 to 40 solar radii) ground based radio scintillation measurements using radio sources (Scott et al. 1983) have shown velocity field fluctuations to increase together with bulk flow speed (both around $\approx 200 \mathrm{~km} \mathrm{~s}^{-1}$ ) but data on correlated magnetic field fluctuations are still missing. Moreover, if one assumes the origin of the MHD fluctuations to lie in photospheric motions at the Sun's surface, one would expect some signature in the observations of a frequency

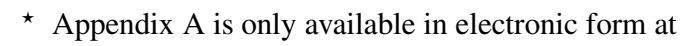
http://www.edpsciences.org corresponding to the characteristic timescale of the energy injection at that level, whereas no such signal is observed. One is then tempted to suppose that strong nonlinear interactions are at work from the very beginning and that the original wave spectrum is modified during propagation through the outer atmosphere by the development of a turbulent cascade. In this way the energy is exchanged between modes of different frequencies and transferred toward smaller scales where dissipation becomes efficient.

The main difficulties one has to face for the development of such a scenario is the nature of the nonlinear interactions for the incompressible Alfvén mode. Among the many wavemodes generated by photospheric oscillations, Alfvén waves are the most likely to survive into the corona (Hollweg 1978), although other modes may be generated throughout the atmosphere. Analysis of observed oscillations in coronal structures may eventually lead to their identification (De Moortel et al. 2002a,b), but we concentrate here on Alfvén waves, since they are the dominant modes observed in the solar wind. It is well known that nonlinear terms couple Alfvén waves propagating in opposite directions. Note, however, that in the fast solar wind, outwardly propagating modes seem to dominate (Smith et al. 1995).

The inhomogeneities of the ambient medium suggest a solution for this apparent contradiction between dominantly unidirectional propagation and development of nonlinear 
interactions (Velli et al. 1989). Variations of the group velocity of the wave (the sum of Alfvén and wind speed gradients) linearly couple the outgoing and ingoing waves producing one from the other and furnishing the trigger for nonlinear interactions to take place. Intensive studies of this mechanism have been carried out in terms of the dynamical time scales which enter the governing equation, while the anisotropic nature of the problem was handled naturally in the context of a Reduced Magneto-Hydrodynamics description (RMHD hereafter) which allows a correct treatment of nonlinear terms (Dmitruk et al. 2001a, 2002; Dmitruk \& Matthaeus 2003; Oughton et al. 2001, 2004). This kind of approach has led to the understanding of the ordering of the characteristic times which should effectively favor the development of a turbulent cascade in planes perpendicular to the direction of wave propagation (along the magnetic field) and the efficiency of dissipation. Due to the complexity of the equations describing the propagation of large amplitude Alfvén waves in an inhomogeneous moving medium these analyses were developed in a static stratified atmosphere where the profiles of the ambient magnetic field and the density of the medium were imposed in order to produce the Alfvén velocity gradients which, ultimately, determine the amount of reflection inside the simulation box.

Other authors (Heinemann \& Olbert 1980; Leroy 1980, 1981; Krogulec et al. 1994; Krogulec \& Musielak 1998; Lou \& Rosner 1994; Mangeney et al. 1991; Grappin et al. 1991; Velli et al. 1991; Velli 1993; Moore et al. 1991; Similon \& Zargham 1992; Cranmer \& van Ballegooijen 2005, but also Dmitruk et al. $2001 \mathrm{~b}$ for a phenomenological nonlinear model) have focused their attention on the linear theory of wave propagation.

Here, three main features have proved to be essential: first, the geometry of the medium, since the profile of Alfvén speed is strongly affected by the structure of the flux tubes in which waves are supposed to propagate; second, the extent of the atmosphere whose global stratification profile determines the transmission, since it is the entire profile of the Alfvén speed, and not simply its local variation, which determines whether or not a wave at a given frequency is capable of escaping at the top of the atmosphere; third, the presence of a wind which separates the atmosphere into two parts. The Alfvén critical point (the distance from the sun at which the wind speed equals the Alfvén speed) represents a natural separation between an internal region where the wind is slow and affects the propagation of the waves only slightly (at least at high frequencies) and an outer region, beyond the critical point, where waves are advected outwards by the wind regardless of their original propagation direction. Even if in the very low corona one might expect the effect of a bulk flow to be negligible, it was shown (Heinemann \& Olbert 1980; Jokipii \& Kota 1989; Velli 1993) that the wind speed reduces the reflection inside the atmosphere for low frequency waves, carrying them out through the Alfvénic critical point (beyond which they can not propagate backwards), and hence enhancing their transmission.

The aim of this paper is to investigate nonlinear effects on wave propagation once the background medium and the entire (lower) atmosphere are taken into account. Following Dmitruk et al. (2001b), we choose a constant or radially expanding transverse dissipative length scale and a phenomenological nonlinear coupling term. This allows us to give a preliminary estimate of dissipation caused by the turbulent cascade of counter-propagating Alfvén waves. Introducing such a term still allows wave propagation to be handled in a relatively simple way and, despite the roughness of the model, allows one to gain insight into the relative importance of the three features listed above when dissipative nonlinear effects are also present.

The paper is structured as follows. First we review the equations which describe Alfvén wave propagation in an inhomogeneous moving medium, then we briefly review the results derived from linear propagation and finally we introduce the phenomenological terms, which split the nonlinear analysis into two models, one in which interactions take place only among waves of the same frequency, and the other in which interactions between different frequencies are taken into account. For parameter space, particular attention is paid to the solar case. Finally we review these results and discuss the development of a more realistic model.

\section{The model}

The equations describing the propagation of Alfvén waves in a non-uniform stationary medium can be derived from the MHD equations under the hypotheses of incompressible adiabatic transverse fluctuations, and after an appropriate averaging over the long timescales of the variation of the solar wind structure (see Velli et al. 1991 for a discussion on the averaging and timescales). The velocity $(\boldsymbol{u})$ and magnetic field fluctuations (b) can be combined to form the Elsässer variables,

$\boldsymbol{z}^{ \pm}=\boldsymbol{u} \mp \frac{\operatorname{sign}\left(\boldsymbol{B}_{0}\right) \boldsymbol{b}}{\sqrt{4 \pi \rho}}$

which describe Alfvén waves propagating outward $\left(z^{+}\right)$or inward $\left(z^{-}\right) . \boldsymbol{B}_{0}$ stands for the average magnetic field (nonuniform) and the sign is taken with respect to the outward direction on the field line while $\rho$ represents the mass density (also non-uniform) of the ambient medium. In terms of these variables the equations for the two fields read (Marsch \& Tu 1989; Zhou \& Matthaeus 1990; Velli 1993):

$$
\begin{aligned}
\frac{\partial z^{ \pm}}{\partial t}+ & {\left[\left(\boldsymbol{U} \pm \boldsymbol{V}_{\mathrm{a}}\right) \cdot \boldsymbol{\nabla}\right] z^{ \pm}+\left(z^{\mp} \cdot \boldsymbol{\nabla}\right)\left(\boldsymbol{U} \mp \boldsymbol{V}_{\mathrm{a}}\right) } \\
+ & \frac{1}{2}\left(z^{-}-z^{+}\right)\left[\boldsymbol{\nabla} \cdot \boldsymbol{V}_{\mathrm{a}} \mp \frac{1}{2}(\boldsymbol{\nabla} \cdot \boldsymbol{U})\right]=-\frac{1}{\rho} \boldsymbol{\nabla} p_{\mathrm{tot}} \\
- & {\left[\left(z^{\mp} \cdot \boldsymbol{\nabla}\right) z^{ \pm}-\left\langle\left(z^{\mp} \cdot \boldsymbol{\nabla}\right) z^{ \pm}\right\rangle\right]+\frac{1}{2}\left(z^{\mp} \cdot \frac{\boldsymbol{\nabla} \rho}{\rho}\right) } \\
& \times\left[\boldsymbol{V}_{\mathrm{a}} \pm \frac{1}{2}\left(z^{-}-z^{+}\right)\right]
\end{aligned}
$$

where $\boldsymbol{U}$ is the average wind speed and the average Alfvén speed is $\boldsymbol{V}_{\mathrm{a}}=\boldsymbol{B}_{0} / \sqrt{4 \pi \rho}$. On the right hand side we have grouped the nonlinear terms including total (magnetic plus gas) pressure, which may be written as the product between $z^{+}$and the gradients of $z^{-}$and viceversa, and the terms involving coupling of fluctuations with gradients of the mean fields. The nonlinear terms which don't average to zero (in angle parenthesis) are to be considered part of the background medium equation, hence must be subtracted in the fluctuation equations. 
When magnetic and gravitational field are collinear, as we assume here throughout, the last terms on the RHS vanishes. In the linear part of the equation we recognize a propagation term (II) and two terms accounting for reflection due to the variation of the properties of the medium, one isotropic (IV) while the other (III) involves variations along the fluctuations' polarization.

The isothermal atmosphere is completely defined by setting the values for temperature, density and magnetic field intensity at the base, together with the mass and radius of the central object $\left(M_{\odot}\right.$ and $\left.R_{\odot}\right)$. The wind speed and Alfvén speed profiles (and their derivatives) depend on the two parameters $\alpha$, the non dimensional scale height, and $\beta_{0}$, the plasma parameter at the base,

$\alpha=\frac{G M_{\odot}}{R_{\odot} c_{\mathrm{s}}^{2}} \approx \frac{v_{\mathrm{esc}}^{2}}{c_{\mathrm{s}}^{2}}$ and $\beta_{0}=\left(\frac{P}{B^{2} / 8 \pi}\right)_{0} \approx \frac{c_{\mathrm{s}}^{2}}{V_{\mathrm{a} 0}^{2}}$,

where $c_{\mathrm{s}}$ is the sound speed and $v_{\mathrm{esc}}$ is the escape speed from the solar surface. This allows one to solve numerically the implicit equation for the isothermal wind,

$\frac{1}{2}\left(\frac{U}{c_{\mathrm{S}}}\right)^{2}-\log \left(\frac{U}{c_{\mathrm{S}}}\right)=2 \log \left(\frac{2}{\alpha} \frac{R}{R_{\odot}}\right)+\alpha \frac{R_{\odot}}{R}-\frac{3}{2}$,

from which the profile for Alfvén speed (as usual $R$ is the heliocentric distance, here $\gamma_{\mathrm{g}}=c_{\mathrm{p}} / c_{\mathrm{v}}$, while $r=R / R_{\odot}$ ) follows:

$\frac{V_{\mathrm{a}}(r)}{c_{\mathrm{s}}}=\frac{1}{r} \sqrt{\frac{2}{\gamma_{\mathrm{g}} \beta_{0}} \frac{U(r)}{U_{0}}} \approx \beta^{-1 / 2}(r)$.

The values at the base for mass density and magnetic field intensity are related by the Alfvén speed definition $\left(\rho_{0}=\right.$ $B_{0}^{2} / 4 \pi V_{\mathrm{a} 0}^{2}$ ), so one has to impose only one of the two, while their profiles are fixed by flux conservation equations,

$\rho(r)=\frac{\rho_{0}}{r^{2}} \frac{U_{0}}{U(r)}, \quad B(r)=\frac{B_{0}}{r^{2}}$.

\subsection{Brief review of linear analysis and results}

Without the nonlinear terms in Eq. (1) the property of wave energy flux conservation (for a static atmosphere) generalizes to conservation of wave action density when a non uniform moving medium is considered (Heinemann \& Olbert 1980; Barkhudarov 1991; Velli et al. 1991). Assuming a temporal dependence of the form $z^{ \pm} \propto \exp [-\mathrm{i} \omega t]$ one finds:

$$
\begin{aligned}
\boldsymbol{\nabla} \cdot\left\{\frac{\rho}{V_{\mathrm{a}}}[\right. & \left(U+V_{\mathrm{a}}\right)\left|z^{+}\right|^{2}\left(\boldsymbol{U}+\boldsymbol{V}_{\mathrm{a}}\right) \\
& \left.\left.-\left(U-V_{\mathrm{a}}\right)\left|z^{-}\right|^{2}\left(\boldsymbol{U}-\boldsymbol{V}_{\mathrm{a}}\right)\right]\right\}=0 .
\end{aligned}
$$

The two distinct fluxes, associated with downward $\left(S^{-} \rightarrow \boldsymbol{U}-\right.$ $\boldsymbol{V}_{\mathrm{a}}$ ) and upward $\left(S^{+} \rightarrow \boldsymbol{U}+\boldsymbol{V}_{\mathrm{a}}\right)$ propagation, in the case of radial propagation reduce to

$S^{ \pm}=F \frac{\left(U \pm V_{\mathrm{a}}\right)^{2}}{U V_{\mathrm{a}}}\left|z^{ \pm}\right|^{2}$,

where $F=\rho U R^{2}$ stands for the total mass flux, also conserved in virtue of the continuity equation; the conservation Eq. (4) thus reduces to the statement $S^{+}-S^{-}=S^{*}=$ const.
It is natural to define the top of the atmosphere at the Alfvénic critical point (labeled with c), since only an outward flux $\left(S_{\mathrm{c}}^{+} \equiv S^{*}\right)$ remains at this point, so that a transmission $(\mathcal{T})$ and a reflection $(\mathcal{R})$ coefficient may be defined:

$\mathcal{T}=\frac{S_{\mathrm{c}}^{+}}{S_{0}^{+}} \equiv \frac{S^{*}}{S_{0}^{+}}$and $\mathcal{R}=1-\mathcal{T}=\frac{S_{0}^{-}}{S_{0}^{+}}$,

where $S_{0}^{+}, S_{0}^{-}$are the fluxes at the base.

Assuming $z^{ \pm}(r, t)=z^{ \pm}(r) \exp [-\mathrm{i} \omega t]$, expressing length in unit of $R_{\odot}$, velocities in units of sound speed, and frequencies in unit of $c_{\mathrm{S}}(\alpha) / R_{\odot}$, the non-dimensional form for Eq. (1), linearized and reduced for the spherical symmetry case, finally becomes:

$$
\begin{aligned}
\frac{\mathrm{d} z^{ \pm}}{\mathrm{d} r} & -\mathrm{i} \frac{\omega}{U \pm V_{\mathrm{a}}} z^{ \pm}+\frac{U \mp V_{\mathrm{a}}}{r\left(U \pm V_{\mathrm{a}}\right)} z^{\mp} \\
& +\frac{1}{2} \frac{z^{-}-z^{+}}{U \pm V_{\mathrm{a}}}\left[\left(2 \frac{V_{\mathrm{a}}}{r} \mp \frac{U}{r}\right)+\frac{\mathrm{d}}{\mathrm{d} r}\left(V_{\mathrm{a}} \mp \frac{1}{2} U\right)\right]=0 .
\end{aligned}
$$

The Alfvén critical point represents a regular singularity for Eq. (7). The regularity conditions for the solutions at this boundary,

$$
\operatorname{Re}\left(z_{\mathrm{c}}^{-}\right)=\frac{\mu \nu}{\mu^{2}+\omega^{2}}\left|z_{\mathrm{c}}^{+}\right| \text {and } \operatorname{Im}\left(z_{\mathrm{c}}^{-}\right)=\frac{\omega v}{\mu^{2}+\omega^{2}}\left|z_{\mathrm{c}}^{+}\right|
$$

can be used to integrate backward to the solar surface. The coefficients $\mu$ and $\nu$ are functions of the Alfvén and wind speed (and their derivatives) calculated at the Alfvénic critical point. In the spherical symmetry case they can be written as

$\mu=\left(\frac{1}{2} \frac{\mathrm{d} U}{\mathrm{~d} r}+\frac{U}{r}\right)_{r=r_{\mathrm{c}}}, \quad v=\left(\frac{1}{2} \frac{\mathrm{d} U}{\mathrm{~d} r}-\frac{U}{r}\right)_{r=r_{\mathrm{c}}}$,

and their values are of the same order of magnitude. It follows that the energy per unit mass for a mode at a given frequency is

$\epsilon_{\mathrm{c}} \equiv \frac{1}{4} \rho_{\mathrm{c}}\left(\left|z_{\mathrm{c}}^{+}\right|^{2}+\left|z_{\mathrm{c}}^{-}\right|^{2}\right)=\frac{1}{4} \rho_{\mathrm{c}}\left|z_{\mathrm{c}}^{+}\right|^{2}\left(1+\frac{v^{2}}{\mu^{2}+\omega^{2}}\right)$.

Finally imposing the same $\left|z_{\mathrm{c}}^{+}\right|$for all the waves one obtains an energy per unit mass which is decreasing with frequency. The transmission coefficient as a function of frequency and temperature is plotted in Fig. 1, as obtained by Velli (1993); the profiles corresponding to $\alpha=4$ and $\alpha=10$ are plotted with thickened lines. As one can see, for all the temperatures considered high-frequency waves $\left(\omega \gtrsim 10^{-3} \mathrm{~Hz}\right)$ are completely transmitted. The behavior at lower frequencies depends on the temperature considered and can be roughly divided into a high and low temperature behavior ( $\alpha \leq 6$ and $\alpha>6$ respectively). For high temperatures the transmission coefficient simply decreases as frequency is decreased and reflection is almost negligible. For low temperatures reflection is stronger and the transmission coefficient reaches a minimum for waves of period around an hour (20\% for $\alpha=15$ ). Lowering the frequency, $\mathcal{T}$ increases and reaches an asymptotic value which is higher for lower temperature. Two main factors produce this behavior in low temperature atmospheres. The first is due to Alfvén speed gradients which are stronger in the low atmosphere, vanish around $R \gtrsim \alpha / 4 R_{\odot}$ and then increase slightly before going asymptotically to zero. This profile produces a tunneling effect for low 


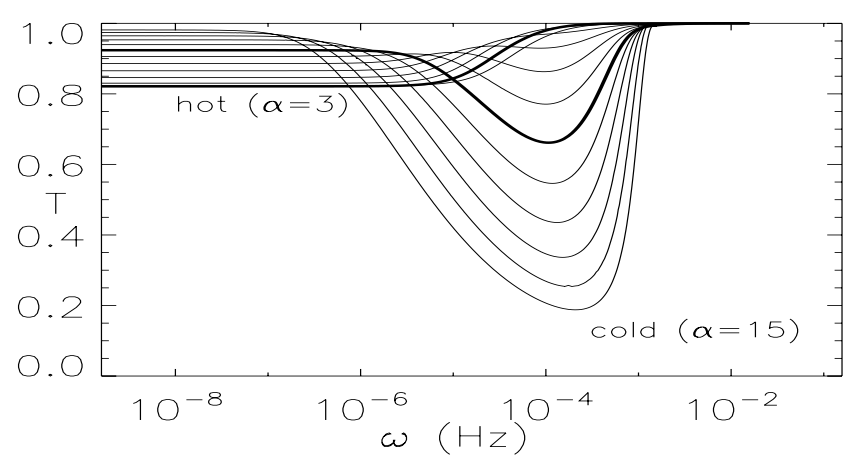

Fig. 1. Transmission coefficient as function of frequency in logarithmic scale for isothermal layers of different temperatures. The value of $\alpha$ ranges from $\alpha=4 \equiv T \approx 3 \times 10^{6} \mathrm{~K}$ (hot) to $\alpha=15 \equiv T \approx$ $8 \times 10^{5} \mathrm{~K}$ (cold) in integer steps. $\beta_{0}=0.08$ for all the profiles (from Velli 1993).

frequencies and it accounts for the increase of $\mathcal{T}$ for decreasing frequencies in the range $5 \times 10^{-6} \mathrm{~Hz} \lesssim \omega \lesssim 5 \times 10^{-4} \mathrm{~Hz}$. The second factor is the presence of a wind, which alters the propagation of waves and carries the low frequency modes $\left(\omega \lesssim 5 \times 10^{-6} \mathrm{~Hz}\right)$ through the critical point: this is what we call the wind effect. From these arguments one would expect low frequency waves to drive the turbulent cascade in hot atmospheres and waves of period of few hours to be the dominant driver in the low temperature atmospheres. However, as we shall see later, the transmission coefficient is an integral quantity, depending on the effect of reflection throughout the whole layer, whereas the local reflection rate plays a more important role in the context of nonlinear interaction and dissipation.

\subsection{A model for nonlinear interaction}

In order to account for nonlinear interactions and turbulent phenomenology we need to add terms on the right hand side of Eq. (7) which contain both of the Elsässer fields and produce a sort of dissipation of the previous linearly conserved quantity $\left(S^{*}\right)$. Following Dmitruk et al. (2001b) we choose the following modeling as substitution for the RHS of Eq. (1),

nonlinear terms $\rightarrow-\frac{z^{ \pm}\left|\boldsymbol{Z}^{\mp}\right|}{L}$

where $L$ represents an integral turbulent dissipation length and $\boldsymbol{Z}^{ \pm}$stands for the total amplitude of the Elsässer field at the point $r$. The nonlinear term contains, a priori, various contributions which come from the different frequencies into which the Alfvén wave packet may be decomposed. The total Elsässer field amplitude is obtained as the root mean squared value of the amplitudes corresponding to the frequencies considered, hence:

$\left|\boldsymbol{Z}^{\mp}\left(\omega_{1}, \omega_{2}, . ., \omega_{n}\right)\right|=\sqrt{\sum_{i}^{n}\left|z^{\mp}\left(\omega_{\mathrm{i}}\right)\right|^{2}}$.

Assuming such a form we will consider interactions among waves with the same frequency (self interacting case), or interactions among a set of waves of different frequencies, in particular we will consider a two frequency case and a three frequency case. As a result the system of equations describing the model changes order (respectively forth, eighth and twelfth). After normalization and time dependence substitution, this leads to the nonlinear model equations,

$$
\begin{aligned}
\frac{\mathrm{d} z^{ \pm}}{\mathrm{d} r} & -\mathrm{i} \frac{\omega}{U \pm V_{\mathrm{a}}} z^{ \pm}+\frac{U \mp V_{\mathrm{a}}}{r\left(U \pm V_{\mathrm{a}}\right)} z^{\mp}+\frac{1}{2} \frac{z^{-}-z^{+}}{U \pm V_{\mathrm{a}}} \\
& \times\left[\left(2 \frac{V_{\mathrm{a}}}{r} \mp \frac{U}{r}\right)+\frac{\mathrm{d}}{\mathrm{d} r}\left(V_{\mathrm{a}} \mp \frac{1}{2} U\right)\right]=-\frac{z^{ \pm}\left|\boldsymbol{Z}^{\mp}\right|}{L\left(U \pm V_{\mathrm{a}}\right)},
\end{aligned}
$$

finally used for numerical integration. (Again $L$ is expressed in units of $R_{\odot}$, velocities in units of $c_{\mathrm{s}}$, and $\omega$ in units of $c_{\mathrm{S}} / R_{\odot}$.)

The form of the nonlinear term may be heuristically derived from the following arguments. When Eq. (1) is Fourier decomposed $\left(z^{ \pm}(\boldsymbol{r}, t) \rightarrow z_{k}^{ \pm}=u_{\boldsymbol{k}} \mp b_{\boldsymbol{k}}\right)$ nonlinear terms couple several wave numbers in the $k$-space. When a strong magnetic field ( $V_{\mathrm{a}}$ in velocity units) is present, the propagation time of the Alfvén waves $\tau_{\mathrm{a}}=\left(\boldsymbol{k} \cdot \boldsymbol{V}_{\mathrm{a}}\right)^{-1}$ is equal or shorter than the characteristic time-scale for nonlinear interaction $\tau_{\mathrm{NL}}=$ $\left(k u_{k}\right)^{-1} \approx\left(k b_{k}\right)^{-1}$ over most of the Fourier space, the nature of the nonlinear cascade is highly anisotropic, developing preferentially in planes perpendicular to the direction of the mean field (Shebalin et al. 1983; Oughton et al. 1994; Goldreich \& Sridhar 1995). It is then useful to decompose local wavenumber in projections along the magnetic field $\left(\boldsymbol{k}_{\|}\right)$and in the perpendicular planes $\left(\boldsymbol{k}_{\perp}\right)$ because energy transfer occurs only among the latter, so that Fourier decomposition is exploited only in $\boldsymbol{k}_{\perp}$. When small fluctuations are considered $\left(V_{\mathrm{a}} \gg b_{k} \approx u_{k}\right)$ these arguments lead to the so called RMHD description which can be derived as an expansion of the usual MHD equations in the small parameter $b_{k} / V_{\mathrm{a}}$ with the restriction $\epsilon_{\mathrm{RMHD}}=\tau_{\mathrm{NL}} / \tau_{\mathrm{a}} \lesssim 1$ (see Oughton et al. 2004 and reference therein for more details on RMHD), in which variations along the perpendicular directions are decoupled from those along the magnetic field $\left(\boldsymbol{\nabla}=\boldsymbol{\nabla}_{\perp}+\boldsymbol{\nabla}_{\|}\right.$, with $\left.\boldsymbol{\nabla}_{\perp} \gg \boldsymbol{\nabla}_{\|}\right)$. We can describe the global effect of this perpendicular cascade by means of two quantities at the large scales, namely an integral scale $\lambda_{0}$, giving the dimension of the greatest eddies in which energy is injected, and the average velocity difference $(\Delta v)$ among points belonging to the same eddy, which in RMHD turbulence also contains magnetic field fluctuations in velocity units $(\Delta b / \sqrt{4 \pi \rho})$. Identifying these two quantities with the integral turbulent length $\left(\lambda_{0}=L\right)$ and the fluctuations' amplitude of the Elsässer fields we can construct a characteristic timescale $\tau_{\mathrm{NL}}^{ \pm}=L /\left|\boldsymbol{Z}^{\mp}\right|$ which accounts for nonlinear turbulent interactions in Eq. (1) (see Dobrowolny et al. 1980 for a more accurate derivation).

Multiplying the above Eq. (12) by the complex conjugate $z^{ \pm *}$ one obtains the evolution equations for the Elsässer energies at a given frequency $E^{ \pm} \equiv \frac{1}{2}\left|z^{ \pm}(\omega)\right|^{2}$. On its RHS one finds $-\left|z^{ \pm}\right|^{2}\left|\boldsymbol{Z}^{\mp}\right| /\left[\left(U \pm V_{\mathrm{a}}\right) L\right]$, which acts as a sink for the energy of a given mode. To quantify the relative importance of various couplings we first consider only nonlinear interactions between counter-propagating waves with the same frequency. Thereafter we consider the more realistic case of different couplings between a fundamental frequency (a very low frequency, sometimes called the quasi-2D component, labeled with index 0 ) and other higher frequencies labeled as interacting (index i).

In both cases, once nonlinear terms are introduced we loose the scaling feature of the linearized equation for which given an 
increment of a factor $f$ in the amplitudes at the critical point we have an equal increment $f$ for the values at the base. Hence, to get realistic values of velocity and magnetic field fluctuations at the base of the atmosphere, we have to tune the amplitude of the outgoing wave imposed at the Alfvénic critical point (since the equations are still integrated backward) for every frequency and coupling considered.

\section{Results}

To quantify dissipation inside the layer one can look at how wave action density changes with $r$ compared to the linear case. It is useful to introduce normalized quantities, i.e. $\mathcal{S}(r) \equiv$ $S^{*}(r) / S^{*}\left(r_{0}\right)$ and what we define to be the dissipation efficiency $\gamma \equiv\left(S_{0}^{*}-S_{\mathrm{c}}^{*}\right) / S_{0}^{*}=1-\mathcal{S}\left(r_{\mathrm{c}}\right)\left(S_{0}^{*} \equiv S^{*}\left(r_{0}\right)\right.$ and $\left.S_{\mathrm{c}}^{*} \equiv S^{*}\left(r_{\mathrm{c}}\right)\right)$. These quantities do not directly yield the amount of energy dissipated, however, the profile of $\mathcal{S}(r)$ shows where the nonlinear dissipative terms have the strongest effect. Further interesting information comes from studying how the values of the Elsässer variables at the base depend on the value at the critical point, departure from a linear scaling being entirely due to nonlinear effects. These amplitudes are constrained by measures of line broadening which give root mean squared values approximately between $20 \mathrm{~km} \mathrm{~s}^{-1}$ and $30 \mathrm{~km} \mathrm{~s}^{-1}$ (Chae et al. 1998) for coronal temperatures.

In the following we first present the results concerning the self-interacting case, where only monochromatic waves interact, then we shall consider modification to dissipation induced by different coupling among two or three frequencies. As model parameters we choose $\alpha \in[4,10]$ corresponding to temperatures for an isothermal layer above the sun's surface ranging from approximately $1 \times 10^{6} \mathrm{~K}(\alpha=10)$ to $3 \times 10^{6} \mathrm{~K}$ $(\alpha=4)$, and we fix the value of the plasma parameter at $\beta_{0}=0.08$ for every temperature; thus, at the base of the atmosphere the Alfvén speed is always five times the sound speed. Finally we set the value of the phenomenological turbulent length $L=0.05 R_{\odot}$, that is about $34000 \mathrm{~km}$ corresponding to the average size of the supergranules at coronal level which is maintained at a constant value through the entire atmosphere.

\subsection{Self-interacting one frequency model}

Consider now nonlinear interactions which couple (only) counter-propagating waves of the same frequency, with the nonlinear terms (NL) having the following form:

$\mathrm{NL} \rightarrow-\frac{z^{ \pm}(\omega)\left|z^{\mp}(\omega)\right|}{L\left(U \pm V_{\mathrm{a}}\right)}$

Although this type of interaction is not dominant (this is a nonresonant interaction unless $\omega$ is so small that $\tau_{\mathrm{NL}}<\tau_{\mathrm{a}}$ ) it is nonetheless a useful step in our modelling in order to understand the behavior of the waves evolution. In Fig. 2, $\mathcal{S}(r)$ is displayed for a high and a low frequency wave $\left(\omega=10^{-2} \mathrm{~Hz}\right.$ and $\omega=10^{-6} \mathrm{~Hz}$ respectively) traveling in $\alpha=4$ and $\alpha=10$ atmospheres, with a reference initial wave amplitude value of $100 \mathrm{~km} \mathrm{~s}^{-1}$ (see Appendix). For the high temperature case (left panel), one obtains what is expected from the linear analysis: high-frequency waves are poorly dissipated while at low
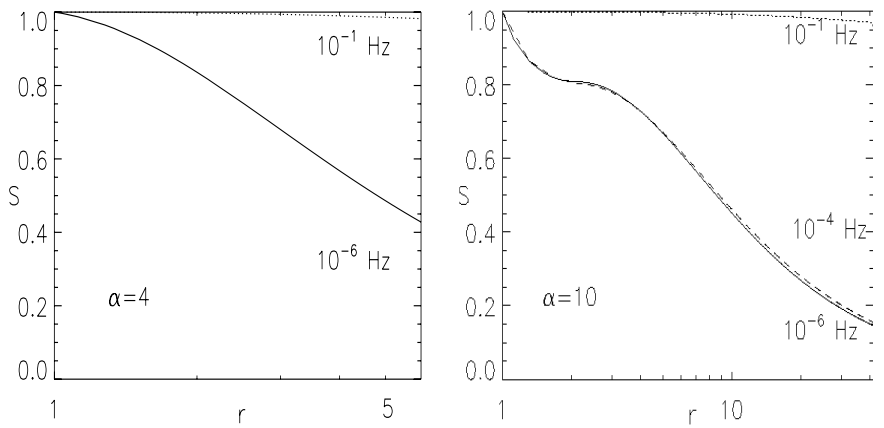

Fig. 2. Total wave action density normalized to the base value as a function of radius in $\alpha=4$ and $\alpha=10$ atmospheres, for a high $\left(10^{-2} \mathrm{~Hz}\right.$, dotted line) and low $\left(10^{-6} \mathrm{~Hz}\right.$, solid line $)$ frequency wave with an initial $100 \mathrm{~km} \mathrm{~s}^{-1}$ wave amplitude. For the cold atmosphere the profile for the wave having the minimum transmission coefficient (dashed line) is also plotted.

frequencies dissipation is enhanced, eventually with more efficiency than one expects on the basis of the transmission coefficient $(\approx 80 \%)$. For low temperatures (right panel), one expects high dissipation for waves with periods of a few hours (transmission $\approx 20 \%$ ) and that is what is found (dashed line), but for the lower frequency waves one finds the same amount of dissipation despite almost perfect linear transmission $(\mathcal{T} \approx 95 \%)$. The reason is that when non-negligible amplitudes are considered, the nonlinear terms dominate over the linear ones where the gradients are strongest and the local reflection rate determines the amount of dissipation. In the right panel, at about $r=\alpha / 4$, dissipation stops and total wave action density remains almost constant. In fact, in this region the reflection rate is negligible as discussed in the linear analysis (in the left panel it coincides with the base of the atmosphere) and no dissipation occurs.

The reflection rate depends both on temperature and wave frequency and it is higher for cooler atmospheres and lower frequency waves. Moreover, most of the reflection takes place in the lower atmosphere, where the Alfvén speed gradients are stronger. For high enough wave amplitudes, nonlinear terms dominate over linear ones and the profile of $\left|z^{-}\right|$is determined uniquely by the local reflection rate, which, for cold atmospheres, is itself dominated by the Alfvén speed gradients.

Ultimately one expects $\gamma\left(\equiv 1-\mathcal{S}_{\mathrm{c}}\right)$ to increase with $\alpha$, and $\mathcal{S}$ to decrease faster with radius in the very low atmosphere. For a given $\alpha$ this behavior should be more pronounced for lowfrequency waves (those suffering stronger reflection) and consequently high-frequency dissipation should be less sensitive to temperature variations. Variation of dissipation efficiency with frequency is shown in Fig. 3 for different initial wave amplitudes $\left(a \rightarrow 100 \mathrm{~km} \mathrm{~s}^{-1}, b \rightarrow 10 \mathrm{~km} \mathrm{~s}^{-1}\right.$ and $\left.c \rightarrow 1 \mathrm{~km} \mathrm{~s}^{-1}\right)$ and both $\alpha=4$ and $\alpha=10$ atmospheres (respectively continuous and dotted lines). One can define a critical frequency $\omega^{*}$ (for which $\gamma$ is half its maximum) that divides the $\gamma(\omega)$ profiles into two branches: a constant low-frequency value and a zero high-frequency one, separated by an intermediate range whose extent depends slightly on temperature and wave amplitude (it decreases with $\alpha$ and $z_{\mathrm{c}}^{+}$). As can be seen in Fig. 3 the value of the critical frequency (marked with a star) depends very little 


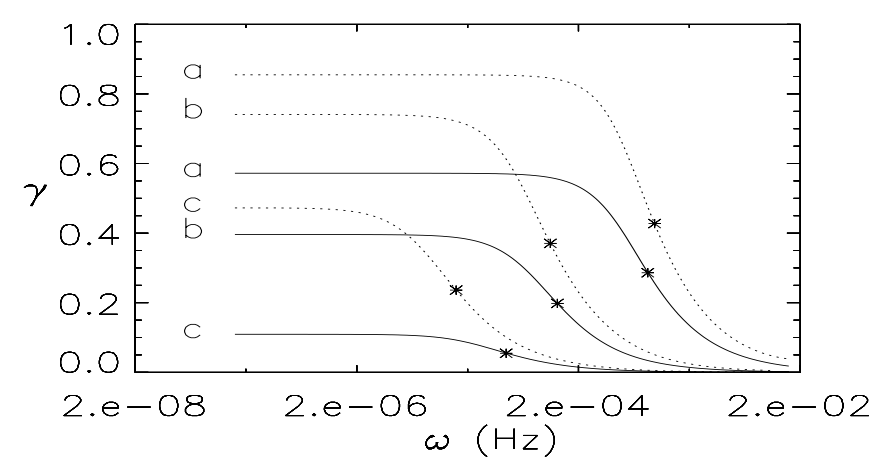

Fig. 3. Dissipation efficiency $(\gamma)$ as function of frequency for a hot ( $\alpha=4$, continuous lines) and a cold ( $\alpha=10$, dotted lines) corona for $\left.\left.z_{\mathrm{c}}^{+}=100 \mathrm{~km} \mathrm{~s}^{-1} \mathbf{a}\right), z_{\mathrm{c}}^{+}=10 \mathrm{~km} \mathrm{~s}^{-1} \mathbf{b}\right)$ and $\left.z_{\mathrm{c}}^{+}=1 \mathrm{~km} \mathrm{~s}^{-1} \mathbf{c}\right)$.

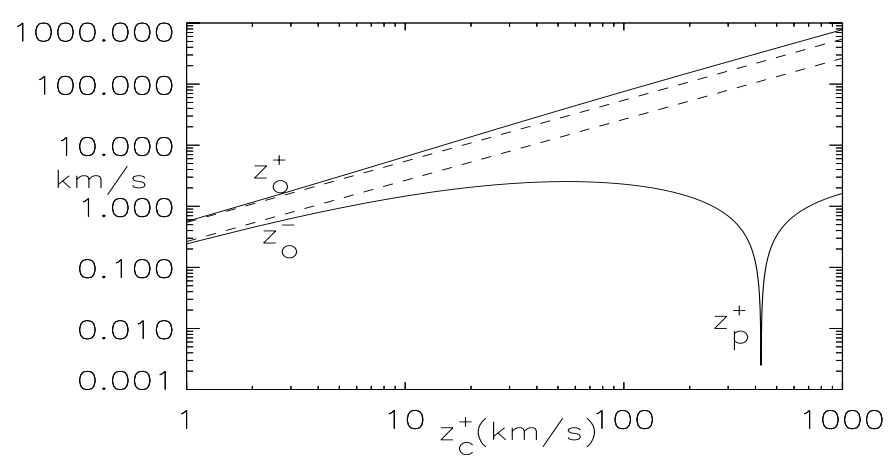

Fig. 4. Outgoing and ingoing wave amplitude (solid line) calculated at the base of the $\alpha=4$ atmosphere, as a function of outgoing amplitude at the critical point. The plots are obtained for $\omega=10^{-6} \mathrm{~Hz}$. Dashed lines show the values obtained in the linear case (the upper and lower line refers to $z^{+}$and $z^{-}$respectively).

on temperature while it seems to scale almost linearly with the value of the amplitude imposed at the top of the atmosphere, indicating that $\left|z_{\mathrm{c}}^{+}\right|$is now a more relevant parameter than temperature. The difference between dissipation efficiency for low and high frequency $(\Delta \gamma)$ depends also on $\alpha$ and the initial wave amplitude and it obviously increases as either parameter is increased.

To investigate the amplitude dependence of the solutions we consider the case of a frequency small enough $(\omega=$ $10^{-6} \mathrm{~Hz}$ ) to remain to the left of $\omega^{*}$ for all temperatures investigated. In Fig. 4 the values of the outgoing and ingoing wave amplitudes at the base are plotted as a function of the outgoing wave amplitude imposed at the critical point, i.e. the energy. The value at the base for the outward propagating waves follows a profile similar to the linear case, it is increased by about the same factor as the initial value at the top of the layer, suggesting that dissipation mostly affects inward propagating waves. As a further indication, their amplitude first grows almost linearly (initial amplitude is not too high and dissipation acts like a perturbation to the linear problem). Then, as $z_{\mathrm{c}}^{+}$further increases, $z_{0}^{-}$decreases and vanishes for a particular value $\left(z_{\mathrm{p}}^{+}\right)$imposed at the critical point. Finally as $\left|z_{\mathrm{c}}^{+}\right|$still grows, $z_{0}^{-}$reaches a very low constant value (say $\left|z_{0}^{-}\right| \approx 10 \mathrm{~km} \mathrm{~s}^{-1}$ ), indicating a kind of saturation which limits inward wave amplitudes at the base. In Fig. 5 dissipation efficiency is plotted as function of initial wave amplitude for

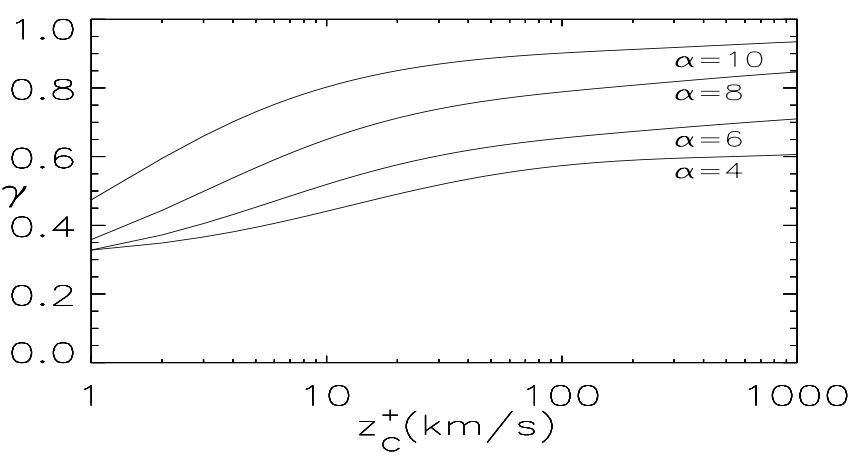

Fig. 5. Dissipation efficiency as function of initial outgoing wave amplitude for $\omega=10^{-6} \mathrm{~Hz}$. The different plots refer to atmospheres with $\alpha=4,6,8,10$.

$\omega=10^{-6} \mathrm{~Hz}$ and $\alpha$ equal to 4, 6, 8 and 10. For low amplitudes $\gamma$ is very low and for the highest two temperatures (negligible reflection) it has the same numerical value. Increasing $z_{\mathrm{c}}^{+}$we arrive to a saturation value depending on temperature (logarithmic scale on $x$ axis) which corresponds to the saturation of the $z^{-}$amplitude at the base of the atmosphere. In the presence of nonlinear interactions it is interesting to remark that the lack of an appreciable reflected wave at the base is not a sign of low dissipation, since as shown above, $z^{-}$may be generated locally and dissipated entirely within the atmospheric layer.

\subsection{Two frequencies interaction}

We use the previous self-interacting analysis in order to identify the interesting couplings among all possible combinations in a set of given representative frequencies. In the following we refer to $\omega_{0}$ as the basic frequency, or fundamental, with which interactions occurs, while with $\omega_{\mathrm{i}}$ we refer to the "interacting" frequencies.

Since in a decaying power law spectrum most of the energy is retained in the low frequencies we expect nonlinear interaction to be more important when such frequencies are involved, hence we choose $\omega_{0}=0$ (representative of low frequencies, say $\omega<\times 10^{-5} \mathrm{~Hz}$ ) and $\omega_{\mathrm{i}}$ varying from about $10^{-5} \mathrm{~Hz}$ to $10^{-2} \mathrm{~Hz}$ increasing by a factor 10 at each step. Specifically, we consider the four couplings,

- a: $\omega_{0}=0 \mathrm{~Hz}-\omega_{\mathrm{i}}=10^{-5} \mathrm{~Hz}$
- b: $\omega_{0}=0 \mathrm{~Hz}-\omega_{\mathrm{i}}=10^{-4} \mathrm{~Hz}$
- c: $\omega_{0}=0 \mathrm{~Hz}-\omega_{\mathrm{i}}=10^{-3} \mathrm{~Hz}$
- d: $\omega_{0}=0 \mathrm{~Hz}-\omega_{\mathrm{i}}=10^{-2} \mathrm{~Hz}$.

As top boundary conditions two cases are considered: a flat spectrum (same energies in the fundamental and interacting modes) and a Kolmogorov-like spectrum, for which the energy per unit mass scales as $\left|z_{\mathrm{c}}^{+}\left(\omega_{\mathrm{i}}\right)\right|^{2}=\left|z_{\mathrm{c}}^{+}\left(\omega_{\text {ref }}\right)\right|^{2} \times\left(\omega_{\mathrm{i}} / \omega_{\text {ref }}\right)^{-2 / 3}$, where $\omega_{\text {ref }}=10^{-6} \mathrm{~Hz}$ is a reference frequency from where the power-law scaling begins $\left(\omega_{\text {ref }} \equiv \omega_{0}\right)$.

Consider first the flat spectrum case shown in Fig. 6 (left panel). The general dissipation profile as a function of $r$ is similar to that formed in Fig. 2 (right panel). The main result here is that higher frequency waves $(b, c, d)$ may also dissipate efficiently thanks to their coupling with the reflected mode of 
A. Verdini et al.: Propagation and dissipation of Alfvén waves in stellar atmospheres permeated by isothermal winds
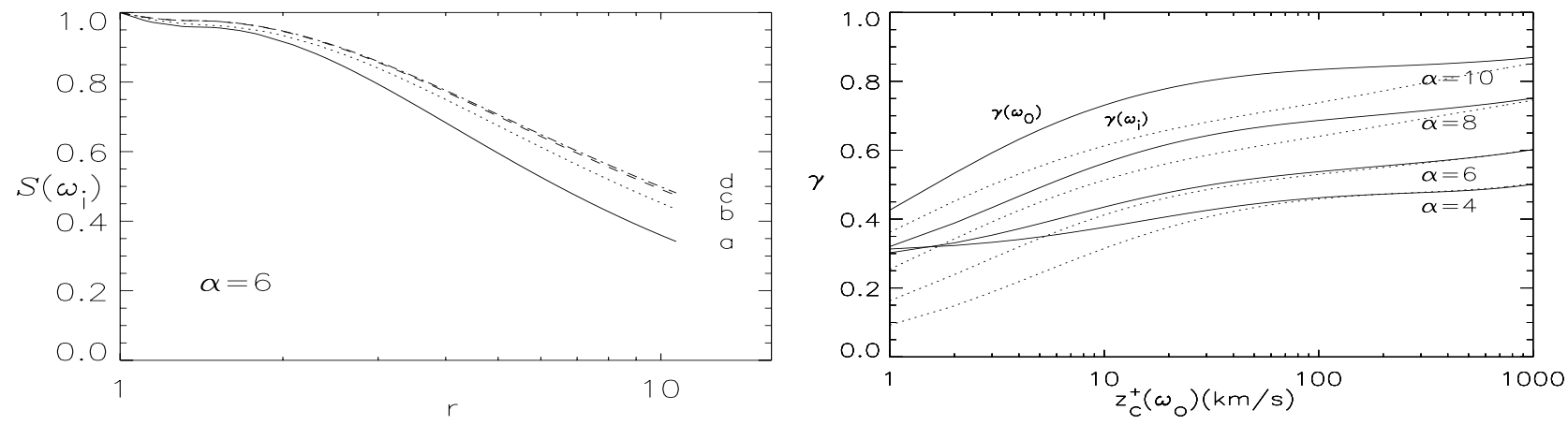

Fig. 6. Flat spectrum. Left panel. Total wave action density, normalized to the base value, for the interacting frequencies in different coupling (labelled with letters, see text), as function of distance from the atmosphere's base expressed in unit of $R_{\odot}$. Temperature is set to $\alpha=6$ and $z_{\mathrm{c}}^{+}\left(\omega_{\text {ref }}\right)=100 \mathrm{~km} \mathrm{~s}^{-1}$. Right panel. Dissipation efficiency as function of initial outgoing wave amplitude $z_{\mathrm{c}}^{+}\left(\omega_{\text {ref }}\right)$. The different plots refer to atmospheres with $\alpha=4,6,8,10$; solid and dotted lines represent respectively $\gamma\left(\omega_{0}\right)$ and $\gamma\left(\omega_{\mathrm{i}}\right)$ with $\left.\omega_{\mathrm{i}}=10^{-2} \mathrm{~Hz}\right)$.
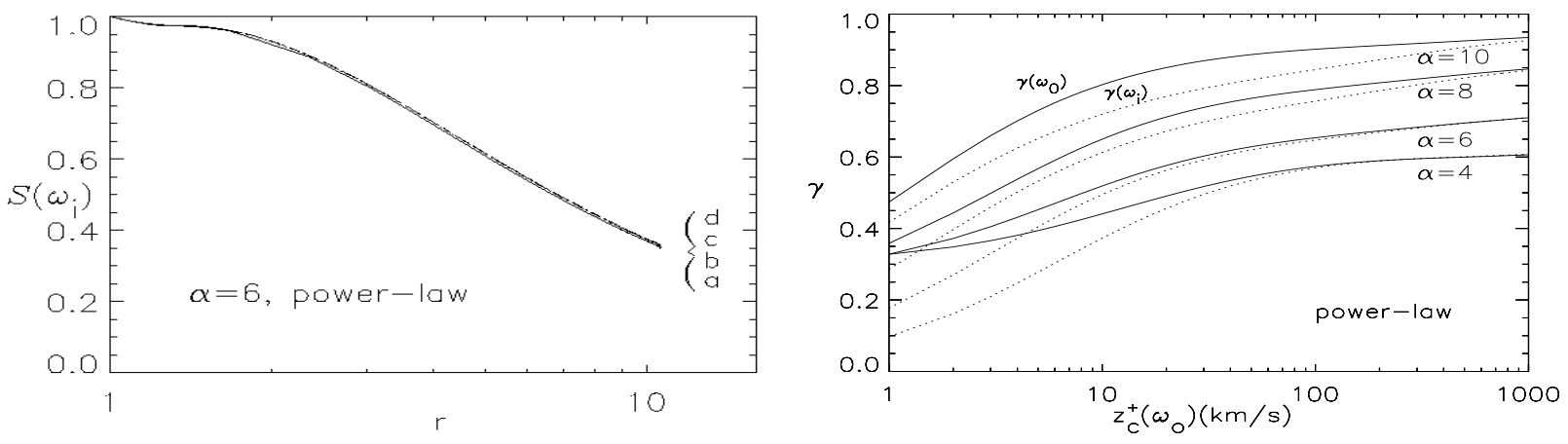

Fig. 7. Same as Fig. 6 but for a power-law spectrum at the critical point.

the very low frequency component, so that all profiles show a significant decrease with distance. This also has an effect on the decay of the lowest (zero) frequency mode, whose reflected mode is ultimately the trigger for nonlinear evolution. When the amplitudes of the higher frequency modes are large (i.e. of the same order of magnitude of the low frequency mode), they influence the evolution of the zero frequency reflected component, which then also affects the zero-frequency outward component, driving the profiles to convergence as illustrated in Fig. 6 (right panel): at low amplitudes $\gamma\left(\omega_{\mathrm{i}}\right)$ differs from $\gamma\left(\omega_{0}\right)$ but at high amplitudes $\gamma\left(\omega_{\mathrm{i}}\right) \approx \gamma\left(\omega_{0}\right)$, which means a strong coupling.

Consider now the power-law spectrum illustrated in Fig. 7. The dissipation profile is the same for all the couplings considered (left panel). Now the energy in the high-frequency waves (mainly propagating outwards) is so small that it has a negligible effect on the evolution of the zero-frequency mode (no back reaction) which, in turn, drives the dissipation of all the coupled modes. On the other hand, in the right panel, one can see that the profiles of $\gamma\left(\omega_{0}\right)$ and $\gamma\left(\omega_{\mathrm{i}}\right)$ are similar to the flat spectrum case and they begin to converge at almost the same values of $z_{\mathrm{c}}^{+}\left(\omega_{0}\right)$.

The two parameters, $\alpha$ and $z_{\mathrm{c}}^{+}\left(\omega_{0}\right)$, determine how strong the coupling is, independently of the shape of the spectrum. For a given amplitude at the critical point of the zerofrequency wave, temperature controls the amount of reflection produced inside the layer, and hence both the linear coupling among the counter-propagating waves of a given frequency (i.e. differences in the waves' evolution due to frequency differences) and the amplitude of the zero-frequency reflected component (the driver). One finds that increasing the temperature (decreasing $\alpha$ ) the coupling becomes weaker for a low $\left|z_{\mathrm{c}}^{+}\right|$and stronger for a high $\left|z_{\mathrm{c}}^{+}\right|$, depending on which of the two above features is dominant. For a given coupling, $\omega_{0}-\omega_{i}$, at a given temperature, the zero frequency wave amplitude imposed at the critical point determines the importance of nonlinear terms (see Fig. 4), and hence the nonlinear coupling among the waves (the evolution independent of frequencies). Increasing $\left|z_{\mathrm{c}}^{+}\left(\omega_{0}\right)\right|$ increases the strength of the coupling.

Two factors determine the rate of dissipation for the coupled waves. The first, as in the self-interacting case, comes from inward propagating wave generation and dissipation. Since this mechanism is driven by reflection it is a characteristic feature of low-frequency waves (the fundamental) and it acts approximately as in the previous analysis, hence it depends most on the low-frequency initial wave amplitude. The second comes from the form chosen for the coupling where high-frequency waves can be dissipated too. They are essentially propagating outward and their main effect is to dissipate the reflected low-frequency wave. The ratio formed with two coupled wave amplitudes controls the relevance of this second aspect. For $z_{\mathrm{c}}^{+}\left(\omega_{\mathrm{i}}\right) \approx z_{\mathrm{c}}^{+}\left(\omega_{0}\right)$, reflected waves are suppressed as soon as they are generated and the dissipation rates of $\mathcal{S}\left(\omega_{\mathrm{i}}\right)$ is increased compared to the self-interacting case but that one of $\mathcal{S}\left(\omega_{0}\right)$ is reduced. When $z_{\mathrm{c}}^{+}\left(\omega_{\mathrm{i}}\right) \ll z_{\mathrm{c}}^{+}\left(\omega_{0}\right)$ the outward highfrequency wave has little capability in suppressing the reflected 
waves and both dissipation rates are almost completely determined by the fundamental mode: no matter what the coupling is, dissipation efficiency is practically the same for the coupled waves (strength of the coupling) and also for all the couplings formed with different interacting frequencies (negligible effect of the high frequency waves).

\subsection{Three frequencies interaction}

Including a third frequency in the nonlinear terms represents not only a simple improvement of the previous two interacting frequencies model but it allows one also to have a rough guide for the evolution of the initial spectrum due to wave propagation. Since the strength of coupling depends both on the temperature of the layer and on the zero-frequency wave amplitude, one expects that modifications of the initial spectrum will be non-negligible only for very low temperature (high $\alpha$ ) or very low initial amplitude. If in fact dissipation efficiency is the same for all the frequencies coupled the spectrum should remain almost unchanged during wave propagation. We shall consider only the power-law case since it better represents the condition of a fully developed turbulent spectrum and the strength of the coupling is not sensitive to the flatness of the spectrum imposed at the top boundary. The energy distribution among frequencies can be imposed by simply specifying the amplitude of the outgoing wave at a given frequency. In fact, as nonlinear terms are introduced, the frequency dependence in Eq. (10) is almost completely removed since $\mu \rightarrow \mu+\left|z_{\mathrm{c}}^{+}\right| / L$. Thus, for high enough initial outgoing amplitude $\mu \gg v$ and $\omega$ so that the contribution of the "ingoing" energy, $\epsilon^{-}$, to the total energy density is negligible.

Supported by these arguments we shall study the behavior of efficiency and spectra with respect to "initial" wave amplitude variation of two representative couplings:

- a: $\omega_{0}=0 \mathrm{~Hz}-\omega_{1}=10^{-2} \mathrm{~Hz}-\omega_{2}=10^{-1} \mathrm{~Hz}$ which involves high-frequency waves,

- b: $\omega_{0}=0 \mathrm{~Hz}-\omega_{1}=10^{-4} \mathrm{~Hz}-\omega_{2}=10^{-2} \mathrm{~Hz}$ which involves intermediate frequency waves lying in the domain of the correlations observed at $1 \mathrm{AU}$.

In the high frequencies coupling (a) the interacting dissipation efficiencies $\left(\gamma\left(\omega_{1}\right), \gamma\left(\omega_{2}\right)\right.$ in the lefthand panel of Fig. 8) practically coincide for all the initial amplitudes considered, while the differences with the fundamental one show again the previously identified dependences: for $\alpha=4$ (and 6) high initial amplitudes (roughly greater than $50 \mathrm{~km} \mathrm{~s}^{-1}$ ) produce strong couplings among the fundamental and interacting modes. However, when we consider cooler atmospheres, only the very high amplitudes (around $1000 \mathrm{~km} \mathrm{~s}^{-1}$ ) are able to equalize the dissipation of all the modes.

For the intermediate frequency coupling (righthand panel), the three curves for the coupled waves follow different profiles and give evidence of how much coupling strength is frequency dependent: for the "middle" frequency (dotted line) dissipation efficiency soon reaches the fundamental mode regime even in the coolest atmospheres, while the highest frequency mode (dashed line) follows almost the same profile as in coupling $a$. A major difference between cool and hot atmospheres
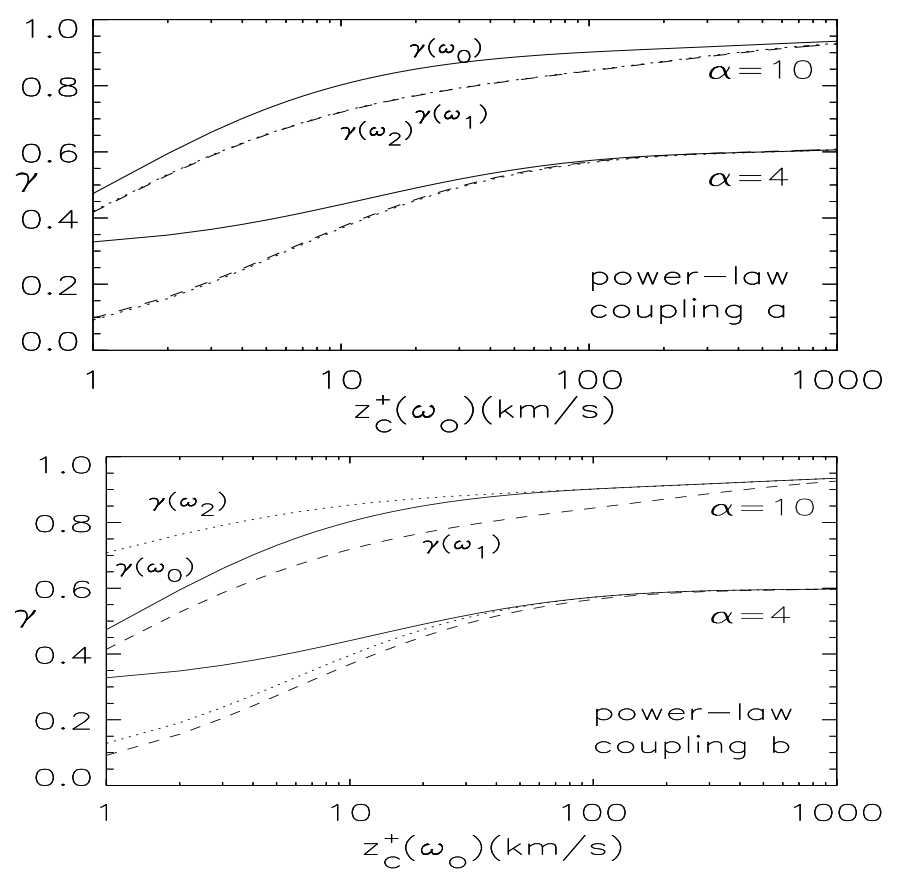

Fig. 8. Dissipation efficiency as function of initial outgoing wave amplitude $z_{\mathrm{c}}^{+}\left(\omega_{0}\right)$ imposed at the top of the layer in the interacting case. The left panel refers to case $a\left(\omega_{1}=10^{-2} \mathrm{~Hz}\right.$ and $\left.\omega_{2}=10^{-1} \mathrm{~Hz}\right)$, and the right panel to case $b\left(\omega_{1}=10^{-4} \mathrm{~Hz}\right.$ and $\left.\omega_{2}=10^{-2} \mathrm{~Hz}\right)$. Initial wave amplitude is scaled following a power-law spectrum (see text). The different plots refer to atmospheres with $\alpha=4,10$; solid, dotted and dashed lines represent respectively $\gamma\left(\omega_{0}\right), \gamma\left(\omega_{1}\right)$ and $\gamma\left(\omega_{2}\right)$.

is the behavior of the middle frequency which, for low enough initial amplitude shows a more efficient dissipation than the fundamental mode. This can be attributed to the wind effect which manifests for cooler atmospheres and weak nonlinear self-interaction or coupling (low amplitudes). It separates very low frequency behavior (wave mainly transmitted, poor dissipation) and intermediate frequency behavior (wave mainly reflected and strong dissipation).

It is then interesting to track the modifications of the imposed (at $r_{\mathrm{c}}$ ) spectra back to the base of the atmosphere, for varying "initial" wave amplitudes. Only coupling $b$ will be considered, for which one expects greater modifications. Figure 9 plots the spectra $\epsilon=\rho\left(\left|z^{+}(\omega)\right|^{2}+\left|z^{-}(\omega)\right|^{2}\right)$ imposed at the top of the atmosphere (dotted lines) and the spectra obtained by integration to the base of the atmosphere (solid lines). Results are shown for two different temperatures $(\alpha=4,10)$. Three representative top-boundary wave amplitudes of the fundamental frequency are considered whose values are $z_{\mathrm{c}}^{+}\left(\omega_{0}\right)=$ $1,100,1000 \mathrm{~km} \mathrm{~s}^{-1}$. Since we impose a power-law spectrum at the top of the layer all dotted lines have slope $-5 / 3$ and can be used as reference to see the modification induced by wave propagation. It is striking how much the spectra remain unchanged for practically all the temperature and all the initial amplitude considered and even if couplings with highest frequency waves are formed (not shown here). With a more accurate inspection one actually finds that the spectra change slightly (see for 

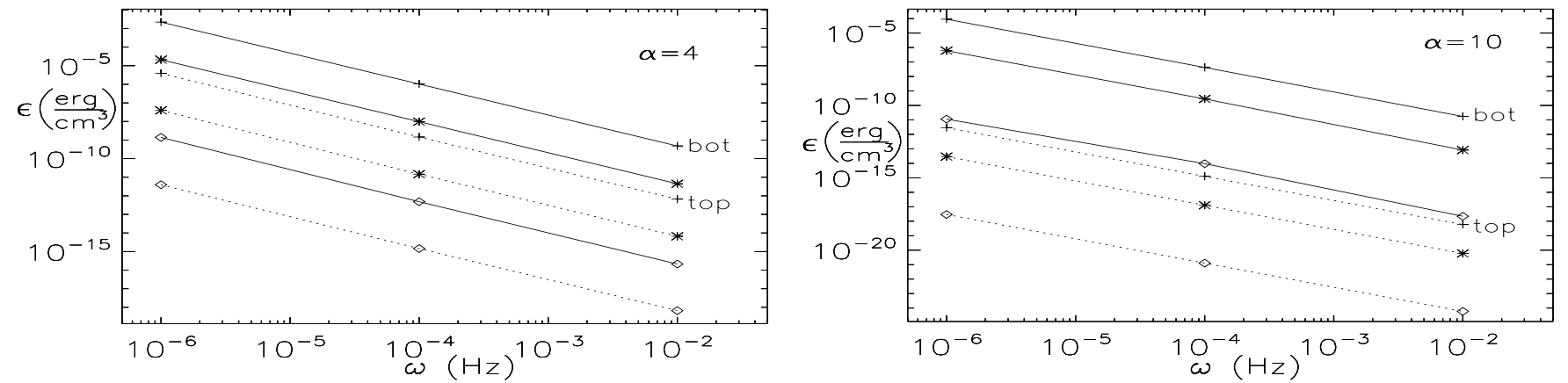

Fig. 9. Spectra at the top (dotted line) and the bottom (solid line) of the atmosphere for three representative initial values of $z_{\mathrm{c}}^{+}\left(\omega_{0}\right)\left(1 \mathrm{~km} \mathrm{~s}{ }^{-1}\right.$, $100 \mathrm{~km} \mathrm{~s}^{-1}$ and $1000 \mathrm{~km} \mathrm{~s}^{-1}$ marked with crosses, stars and diamonds respectively) and different temperature $(\alpha=4,10$ respectively on the left and right panel) in coupling $b$.
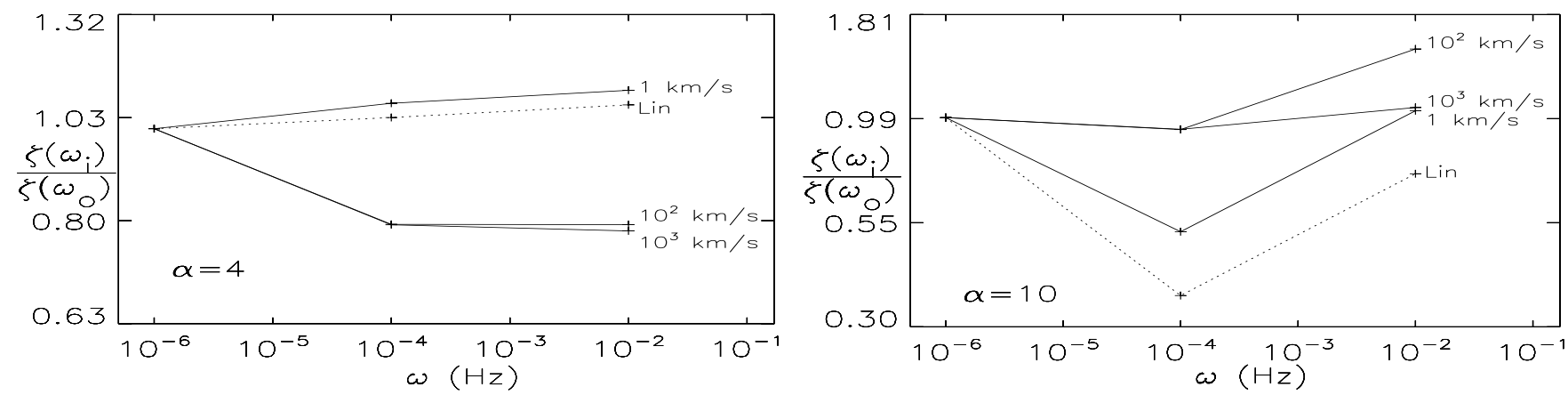

Fig. 10. Ratio $\xi$ between the spectra at the top and the bottom of the atmosphere for $\alpha=4,10$ (respectively left and right panel) in the case of coupling $b$. The plots are labeled with the initial amplitudes $z_{\mathrm{c}}^{+}\left(\omega_{0}\right)$ imposed at the top of the layer. Also shown is the ratio for the linear case (dotted line).

example solid the line marked with diamonds in the right panel). The ratio

$\xi(\omega)=\frac{\epsilon_{\mathrm{c}}(\omega)}{\epsilon_{0}(\omega)}=\frac{\rho_{\mathrm{c}}\left(\left|z_{\mathrm{c}}^{+}(\omega)\right|^{2}+\left|z_{\mathrm{c}}^{-}(\omega)\right|^{2}\right)}{\rho_{0}\left(\left|z_{0}^{+}(\omega)\right|^{2}+\left|z_{0}^{-}(\omega)\right|^{2}\right)}$

is then introduced to highlight the very small differences among these cases.

In Fig. 10 we plot such ratios normalized to the value of the fundamental mode, i.e. $\xi\left(\omega_{1}\right) / \xi\left(\omega_{0}\right)$ and $\xi\left(\omega_{2}\right) / \xi\left(\omega_{0}\right)$ (the fundamental being normalized to its value) for the cases shown in Fig. 9. Since the spectrum at the top is fixed by the initial condition, a normalized $\xi$ lower (greater) than one means the spectrum is steepening (flattering).

For low initial amplitudes in hot atmospheres the spectrum becomes flatter at high frequencies, since the coupling is not so strong and frequency dependence displays its influence. As we increase the strength of the coupling, by increasing $z_{\mathrm{c}}^{+}\left(\omega_{0}\right)$, differences are smoothed and the spectrum first adjusts itself to the slope imposed at the top of the atmosphere and then finally steepens. The same behavior is observed for the higher frequency coupling case $a$ (not shown), so one can attribute such a general feature to the high temperature atmospheres.

For low temperatures, the picture is more complicated. Frequency differences are more important and the strength of the coupling is reduced, the intermediate frequency waves contribute to dissipation with their self-interacting part so that the spectrum steepens at intermediate frequencies and flattens at high frequency. To quantify this tendency, we have calculated the exponent of the resulting power-law scaling at the base for the low and high frequency branches. In the worst of the cases $\left(1 \mathrm{~km} \mathrm{~s}^{-1}\right.$ for $\alpha=10$ in Fig. 10) the slopes are 1.61 (spectrum steepening) and 1.76 (spectrum flattening) to compare with 1.667 , but generally the slope is 1.65 (steepening) or 1.67 (flattering). One can then conclude that even if the spectra evolves during propagation the differences between the slopes at the top and bottom are always very small or even negligible.

Note how for lower $\alpha$ and low amplitude the spectrum is unchanged by propagation, even though consistent differences in the dissipation efficiencies of the coupled modes are found. The origin of such discrepancies is to be found in the definition of $\gamma$ which involves total wave action density at the boundaries of the layer, including a contribution from inward and outward fluxes at the boundaries which are not simply wave amplitudes (which indeed determine the spectrum). One can in fact rewrite $\gamma$ as follows:

$$
\begin{aligned}
\gamma(\omega)= & 1-\frac{S\left(r_{\mathrm{c}}\right)}{S\left(r_{0}\right)} \\
= & 1-\frac{\rho_{\mathrm{c}} U_{\mathrm{c}} R_{\mathrm{c}}^{2}\left[\left(U_{\mathrm{c}}+V_{\mathrm{ac}}\right)^{2}\left|z_{\mathrm{c}}^{+}\right|^{2}\right]}{U_{\mathrm{c}} V_{\mathrm{ac}}} \\
& \times \frac{U_{0} V_{\mathrm{a} 0}}{\rho_{0} U_{0} R_{0}^{2}\left[\left(U_{0}+V_{\mathrm{a} 0}\right)^{2}\left|z_{0}^{+}\right|^{2}-\left(U_{0}-V_{\mathrm{a} 0}\right)^{2}\left|z_{0}^{-}\right|^{2}\right]} \\
= & 1-g(\alpha) \frac{\left|z_{\mathrm{c}}^{+}\right|^{2}}{\left|z_{0}^{+}\right|^{2}-\left|z_{0}^{-}\right|^{2}[1-g(\alpha)]},
\end{aligned}
$$


where $g(\alpha)=4 U_{0} V_{\mathrm{a} 0} /\left(U_{0}+V_{\mathrm{a} 0}\right)^{2}$. In particular $U_{0} / V_{\mathrm{a} 0}<1$ for all temperatures considered and tends to zero as $\alpha$ is increased (so that $g \approx 4 U_{0} / V_{\mathrm{a} 0} \rightarrow 0$ ). Since the contribution of the energy of the inward waves, $\epsilon^{-}$, at the top boundary is always negligible, if $\left|z_{0}^{-}\right| \ll\left|z_{0}^{+}\right|$(cf. saturation of $\left|z_{0}^{-}\right|$in Fig. 4), one can finally write:

$\gamma \approx 1-g(\alpha) \frac{\left|z_{\mathrm{c}}^{+}\right|^{2}}{\left|z_{0}^{+}\right|^{2}} \times\left[1+\frac{\left|z_{0}^{-}\right|^{2}}{\left|z_{0}^{+}\right|^{2}}(1-g(\alpha))\right]$,

$\xi \approx \frac{\rho_{c}}{\rho_{0}} \frac{\left|z_{\mathrm{c}}^{+}\right|^{2}}{\left|z_{0}^{+}\right|^{2}} \times\left[1-\frac{\left|z_{0}^{-}\right|^{2}}{\left|z_{0}^{+}\right|^{2}}\right]$

with the terms in square brackets approximately equal to one. For a given temperature, a difference in the $\gamma(\omega)$ signifies a real difference in the $\xi(\omega)$ only if the contribution of the inward reflected wave amplitude at the base of the layer is negligible. For low amplitudes (weak coupling) the reflected waves of the fundamental mode have still a considerable amplitude at the base (see Fig. 4) and this approximation is no longer valid. Moreover, for a high temperature atmosphere $g(4) \approx 0.26$, and the difference $\gamma\left(\omega_{0}\right)-\gamma\left(\omega_{\mathrm{i}}\right)$ is enhanced with respect to those occurring in the lower temperature atmospheres, exaggerating the differences in the waves evolution.

\subsubsection{The effect of a radially expanding turbulent scale}

Up to now we have considered a fixed turbulence scale, $L$, in a wind which is spherically expanding. This assumption produces strong consequences on the dissipation rate in the higher part of the atmosphere and hence on dissipation efficiency (Zank et al. 1996; Matthaeus et al. 1998). In that region, in fact, Alfvén speed and wind speed gradients are weaker and we expect only a small amount of reflection. However, if the turbulence scale is held constant, the phenomenological gradient $(\sim 1 / L)$ becomes more and more important as we move into the outer atmosphere since it is not expanding as the other gradients or length-scales in Eq. (12) are. Hence the nonlinear term increases and the net result is an enhancement of dissipation, especially for lower temperature atmospheres which extend up to about $40 R_{\odot}$. In order to correct such effects and to consider a consistent spherical expansion, we modify the three frequencies model to use $L=L_{0} \times r$, with $L_{0}$ fixed at the coronal level by the average dimension of the supergranules.

For low $z_{\mathrm{c}}^{+}\left(\omega_{0}\right)$, dissipation efficiency is considerably altered (reduced) with respect to the non-expanding case (compare Figs. 11 and 8). Since nonlinear term influences are now reduced, we need greater initial amplitudes to efficiently couple the frequencies considered; as a consequence the profiles of $\gamma$ are shifted to the right with respect to the non-expanding model (by a factor of 3 or 4 ). For coupling $a$, the plots of $\gamma$ show the same kind of variations as discussed above and the profile of the interacting frequencies $\left(\omega_{i 1}\right.$ and $\left.\omega_{i 2}\right)$ again coincides for every amplitude and temperature. Their values are identical to the high frequency one in coupling $b$ (dashed lines in Fig. 11).

If we again construct the ratio $\xi$, then when we consider high temperatures we obtain the same features as in the nonexpanding case, but in the "worst cases" (low temperature and

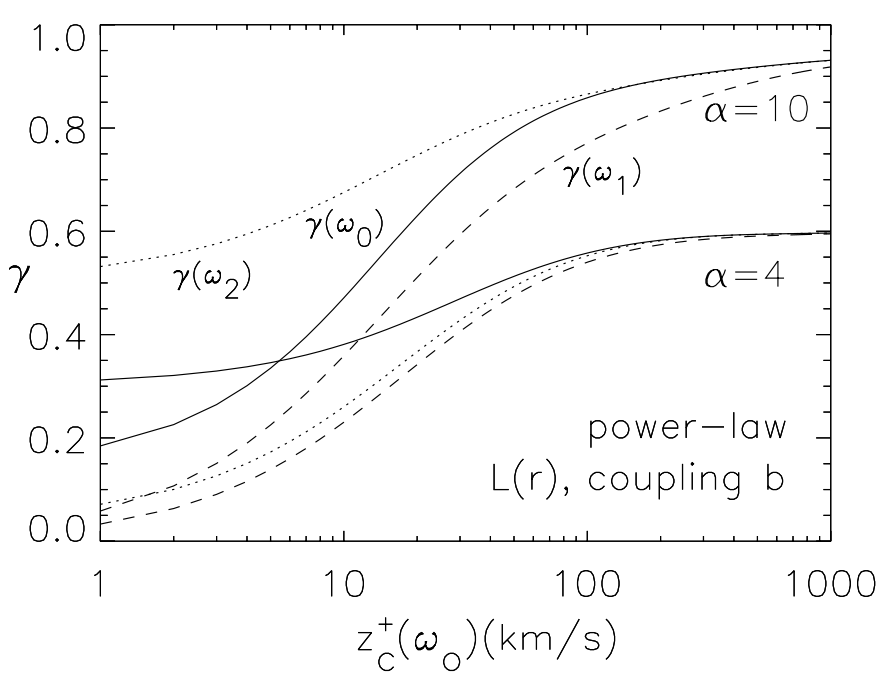

Fig. 11. Same as in Fig. 8 for coupling $b$ and power-law initial spectrum except that integrations are performed using a spherically expanding turbulent length $L(r)=L_{0} \times r$ with $L_{0}$ fixed by the average dimension of supergranules at coronal level. Results are shown for $\alpha=4,10$ atmospheres.

low amplitudes) differences in the slopes are more evident suggesting some spectral in such conditions.

\section{Conclusions}

In this paper we have modeled the nonlinear evolution of Alfvén waves propagating through the subAlfvénic region of isothermal stellar winds. Nonlinear interactions occur between outward propagating and reflected waves, and it is assumed that a nonlinear cascade develops preferentially in a direction perpendicular to that of propagation, which we take to coincide with the direction of the mean radial magnetic field. The nonlinear term then acts as a dissipative sink for both outward and inward waves of a given frequency.

As might be expected, lower temperature atmospheres, with higher gradients, and lower frequency waves allow a stronger dissipation of outwardly propagating waves. On the other hand our results seem to imply that a well developed turbulent spectrum does not change appreciably during propagation.

The dissipation rate has been studied, varying the temperature of the layer and the frequency and amplitude (imposed at the critical point) of the waves. We find that for a given amplitude and frequency, the dissipation rate is stronger in the lower part of the atmosphere and depends strongly on the temperature, which ultimately determines the amount of reflection via the density gradients. As the wave amplitude is increased, the dissipation rate is enhanced, reaching a saturation value which depends on temperature and frequency. Saturation is an effect of nonlinear-dissipative interactions which limit the inward propagating (reflected) wave amplitude once the outgoing amplitude is increased beyond a given value (which also depends on temperature and frequency). Below a critical frequency (whose temperature dependence is negligible), dissipation assumes a constant rate all the way down to "zero-frequency" 
fluctuations, while above the critical frequency the dissipation rate tends to zero as frequency is increased, since the amount of reflection decreases with frequency (WKB behavior). The local interaction analysis shows that even if the amplitudes of the inward propagating waves at the base are negligible, continuous reflection due to wind and Alfvén speed gradients can produce significant dissipation (see comments in Fig. 5 at the end of Sect. 3.1); however, only low frequency waves are efficiently dissipated for reasonable wave amplitudes.

We have also investigated a more realistic calculation, considering non-local interactions, where an outwardly propagating Alfvén wave is allowed to interact nonlinearly with the total rms value of inward fluctuations summed over all frequencies (and vice versa). Our approach differs from what has been done in a recent paper of Cranmer \& van Ballegooijen (2005) in two related aspects concerning the nonlinear-dissipative interactions. We consider the full expression of the wave action density, hence including the downward propagating flux, or in other words, the nonlinear equations of the Elsässer fields are integrated simultaneously to include the effect of the dissipation in a consistent way. Nonlinear interactions act on both types of (counterpropagating) waves, thereby producing the attenuation of the reflected component which affects the driving of the global dissipation. In our analysis, the description of the total rms value of the fluctuation is approximated by two or three representative waves with different frequencies spread across a spectrum, the "zero frequency" wave for the low frequencies, and the higher frequencies selected from spectra with different slopes. The strength of the coupling, i.e., the total energy in the outward modes at the lower frequencies (for which reflection is efficient) is crucial for the way in which energy is dissipated along the spectrum. If the amplitude of the low frequency wave is high enough (say $10 \mathrm{~km} \mathrm{~s}^{-1}$ at the base for a $10^{6} \mathrm{~K}$ atmosphere) dissipation of all the outward modes is driven by the low-frequency (quasi-2D) reflected waves: the coupling may be considered strong. A second important aspect is the slope of the spectrum or equivalently the relevant energy residing in the higher frequency waves. Its effect is to enable dissipation of the low-frequency reflected component, since there is little reflected energy at high frequencies, and ultimately to reduce the dissipation efficiency of all the waves coupled. In summary, for a given total outward energy, dissipation is more efficient if the spectra have higher energies at lower frequencies, i.e. steeper spectral slopes.

Setting the lowest frequency to higher values, say $\omega_{0}=$ $10^{-5} \mathrm{~Hz}$, produces some differences in the results, but the global analysis remains unchanged. From Fig. 3 one can guess how the strength of the coupling is affected and hence how the spectra change. In fact the higher the frequency is, the lower the amount of reflected waves; hence the lowest frequency wave is less efficient in driving the dissipation of all the waves coupled. Imposing the same amplitude at the critical point the strength of the coupling is lower, linear effects (i.e. differences in the wave propagation due to different reflection rates) become more important and the spectra show a somewhat higher modification. However, increasing the amplitude of the lowest frequency wave restores the importance of nonlinear terms (the coupling) which overcome the linear effects. These considerations remain valid if one chooses the lowest frequency in the low-frequency plateaus of the curves in Fig. 3. Note that the rightward extension of the plateau increases with increasing wave amplitude.

We have also considered a spherically expanding length scale $L(r)=L_{0} \times r$. In this case, the qualitative features discussed above remain essentially the same (for high enough initial amplitudes). The main effect of the expansion is to reduce the phenomenological gradient $1 / L(r)$ entering the nonlinear term of Eq. (12) as we move further out in the atmosphere so that dissipation in the higher part of the layer is greatly reduced. As a result the amount of energy dissipated is decreased and one can actually attribute differences in the dissipation rate of waves at different frequencies almost entirely to differences in their propagation through the low atmosphere where gradients are greater and hence where most of reflection takes place.

Of course, to better understand the spectral evolution in a stratified atmosphere one should also include the chromosphere, the photosphere and the transition region. Here different physical conditions are encountered and it is not clear how the evolution of the waves in these regions affects the development of "Alfvénic turbulence".

Modeling the deeper stratified layers as a set of isothermal layer with different temperatures (with a discontinuity across the transition region) produces changes of the parameter $\beta$ which has been held fixed in our analysis. In our case, how changes in $\beta$ affect the result may be discussed qualitatively. First note that for a given temperature, the wind solutions are selected (see Eq. (2)) and hence the wind profile and its gradients remain the same. As $\beta$ is increased the Alfvén speed decreases (still maintaining its characteristic profile, i.e. a maximum at $\alpha / 4$ ), its gradients decrease too and the Alfvén critical point moves to lower radii. In the linear case the net effect is an enhanced transmission at low and intermediate frequencies. When nonlinear interactions are taken into account the amplitudes must be scaled to get the same quantitative results, i.e. the same amplitude may be regarded as low in a high $\beta$ plasma or high in a low $\beta$ plasma as far as the strength of the coupling is concerned because, for a given temperature, the strength depends entirely on the amount of reflection. When multiple isothermal layers with different temperatures and different thicknesses are considered, other characteristic length scales and gradients are introduced in the equation describing the wave propagation. The transmission properties of the entire atmosphere are altered, as are the properties of wave dissipation and the spectral slope (we leave this question to a following paper).

Our results concern the evolution of a spectrum formed with only three frequencies which might not capture the whole shape modification in a complex atmosphere. In our model atmosphere, even if highly stratified, the density gradients, change gradually without discontinuities. However, the Alfvén waves observed in the solar wind do not necessarily originate at a photospheric level and can be generated directly in the corona. Moreover coronal structures can produce localized gradients so that the propagation of the waves can be dramatically altered with respect to the simple case studied here. One can then say that many processes, different 
from a turbulent cascade, are able to transform a complex signal into a simple spectrum as observed in situ in the solar wind.

Acknowledgements. We acknowledge financial assistance from the European RT network TOSTISP contract No. HPRN-CT-200100310 and from the Italian Ministry of Research contract COFIN No. 2002025872, and the New Zealand Marsden Fund (02-UOW-050 MIS). We would like to thank the IPAM program "Great Challenge Problems in Computational Astrophysics" at UCLA where this work was completed. A. Verdini would like to thank S. Landi for carefully reading the manuscript and for useful suggestions.

\section{References}

Barkhudarov, M. R. 1991, Sol. Phys., 135, 131

Chae, J., Yun, H. S., \& Poland, A. I. 1998, ApJS, 114, 151

Cranmer, S. R., \& van Ballegooijen, A. A. 2005, ApJS, 156, 265

De Moortel, I., Hood, A. W., Ireland, J., \& Walsh, R. W. 2002a, Sol. Phys., 209, 89

De Moortel, I., Ireland, J., Walsh, R. W., \& Hood, A. W. 2002b, Sol. Phys., 209, 61

Dmitruk, P., \& Matthaeus, W. H. 2003, ApJ, 597, 1097

Dmitruk, P., Matthaeus, W. H., Milano, L. J., \& Oughton, S. 2001a, Physics of Plasmas, 8, 2377

Dmitruk, P., Milano, L. J., \& Matthaeus, W. H. 2001b, ApJ, 548, 482

Dmitruk, P., Matthaeus, W. H., Milano, L. J., et al. 2002, ApJ, 575, 571

Dobrowolny, M., Mangeney, A., \& Veltri, P. 1980, Phys. Rev. Lett., 45,144

Goldreich, P., \& Sridhar, S. 1995, ApJ, 438, 763

Grappin, R., Velli, M., \& Mangeney, A. 1991, Annales Geophysicae, 9,416

Heinemann, M., \& Olbert, S. 1980, J. Geophys. Res., 85, 1311

Hollweg, J. V. 1978, Geophys. Res. Lett., 5, 731
Hollweg, J. V., Jackson, S., \& Galloway, D. 1982, Sol. Phys., 75, 35

Jokipii, J. R., \& Kota, J. 1989, Geophys. Res. Lett., 16, 1

Krogulec, M., \& Musielak, Z. E. 1998, Acta Astron., 48, 77

Krogulec, M., Musielak, Z. E., Suess, S. T., Nerney, S. F., \& Moore, R. L. 1994, BAAS, 26, 1472

Leroy, B. 1980, A\&A, 91, 136

Leroy, B. 1981, A\&A, 97, 245

Lou, Y., \& Rosner, R. 1994, ApJ, 424, 429

Mangeney, A., Grappin, R., \& Velli, M. 1991, Advances in Solar System Magnetohydrodynamics, ed. E. R. Priest, \& A. W. Hood, 327

Marsch, E., \& Tu, C. 1989, J. Plasma Phys., 41, 479

Matthaeus, W. H., Smith, C. W., \& Oughton, S. 1998, J. Geophys. Res., 103, 6495

Matthaeus, W. H., Zank, G. P., Oughton, S., Mullan, D. J., \& Dmitruk, P. 1999, ApJ, 523, L93

Moore, R. L., Suess, S. T., Musielak, Z. E., \& An, C.-H. 1991, ApJ, 378,347

Oughton, S., Priest, E. R., \& Matthaeus, W. H. 1994, J. Fluid Mech., 280,95

Oughton, S., Matthaeus, W. H., Dmitruk, P., et al. 2001, ApJ, 551, 565

Oughton, S., Dmitruk, P., \& Matthaeus, W. H. 2004, Phys. Plasmas, 11,2214

Scott, S. L., Coles, W. A., \& Bourgois, G. 1983, A\&A, 123, 207

Shebalin, J. V., Matthaeus, W. H., \& Montgomery, D. 1983, J. Plasma Phys., 29, 525

Similon, P. L., \& Zargham, S. 1992, ApJ, 388, 644

Smith, E. J., Balogh, A., Neugebauer, M., \& McComas, D. 1995, Geophys. Res. Lett., 22, 3381

Velli, M. 1993, A\&A, 270, 304

Velli, M., Grappin, R., \& Mangeney, A. 1989, Phys. Rev. Lett., 63, 1807

Velli, M., Grappin, R., \& Mangeney, A. 1991, Geophys. Astrophys. Fluid Dynamics, 62, 101

Zank, G. P., Matthaeus, W. H., \& Smith, C. W. 1996, J. Geophys. Res., 101,17093

Zhou, Y., \& Matthaeus, W. H. 1990, J. Geophys. Res., 95, 10291 
A. Verdini et al.: Propagation and dissipation of Alfvén waves in stellar atmospheres permeated by isothermal winds, Online Material $p 1$

\section{Online Material}




\section{Appendix A: Realistic wave amplitudes}

As mentioned at the end of Sect. 2.2, non-linear models do not have a scaling normalization factor as linear ones do, so to have an idea of the realistic values of wave amplitude to be imposed at the top of the layer we have calculated, for different temperatures and the different models explored, the outgoing wave amplitude $z_{\mathrm{c}}^{+}$which results from an rms velocity field fluctuation at the coronal base ranging from $20 \mathrm{~km} \mathrm{~s}^{-1}$ to $30 \mathrm{~km} \mathrm{~s}^{-1}$. Now quantitative differences among the models considered (especially in the case with turbulent scale length expansion) become important. In Table A.1 we display the amplitudes at the top of the layer for various frequencies and coupling models which yield the "realistic" amplitudes at the coronal base. Since the equations are integrated backward, the energy we impose at the critical point decreases as the dissipation increases. In the first box, base values are given for the local wave interaction model; in the second box base values are given for the global interaction model with a flat spectrum, and finally in the third box we display the results obtained when a power-law initial spectrum is imposed at the top of the atmosphere. Presence of an expanding turbulent length is specified directly in the table (labeled with an $L(r)$ ).

Note how the differences in the amount of dissipated energy among different couplings (second box) disappear as the power-law scaling is introduced in the initial (top) condition (compare the values relative to cases I, II, III and IV with those ones in the last box). Generally we can say that for lower temperature and lower frequencies (of the coupled waves) the dissipation is higher. Comparing again the results obtained when flat or power-law spectra are imposed as the initial $\left(r=r_{\mathrm{c}}\right)$ condition, we can conclude that in the former dissipation efficiency is stronger (Fig. 7 right panel) even if the total amount of dissipation is actually reduced (because the flat spectrum contains a greater amount of energy to start with). 
A. Verdini et al.: Propagation and dissipation of Alfvén waves in stellar atmospheres permeated by isothermal winds, Online Material $p 3$

Table A.1. Values of the outgoing wave amplitude $\left(\mathrm{km} \mathrm{s}^{-1}\right)$ imposed at the top of the atmosphere which, once integrated backward, give rms velocity field fluctuations values at the base between $20 \mathrm{~km} \mathrm{~s}^{-1}$ and $30 \mathrm{~km} \mathrm{~s}^{-1} . L(r)$ signifies that an expanding turbulent lengthscale has been used. Except where indicated a flat spectrum is imposed at the top boundary.

\begin{tabular}{ccccc}
\hline \hline Frequencies or & \multicolumn{3}{c}{$\left|\mathbf{z}_{\mathbf{c}}^{+}\right|\left(\mathrm{km} \mathrm{s}^{-1}\right)$ giving $u_{0}=[20-30] \mathrm{km} \mathrm{s}^{-1}$ for } \\
couplings & $\alpha=4$ & $\alpha=6$ & $\alpha=8$ & $\alpha=10$ \\
\hline $10^{-6} \mathrm{~Hz}$ & $54-80$ & $85-126$ & $131-191$ & $187-272$ \\
$10^{-4} \mathrm{~Hz}$ & $54-80$ & $86-126$ & $131-191$ & $187-272$ \\
$10^{-3} \mathrm{~Hz}$ & $58-83$ & $89-129$ & $135-195$ & $192-278$ \\
$10^{-2} \mathrm{~Hz}$ & $76-110$ & $126-178$ & $215-293$ & $353-464$ \\
\hline $10^{-6} \mathrm{~Hz}-10^{-2} \mathrm{~Hz} ; \rightarrow \mathrm{I}$ & $43-63$ & $70-102$ & $114-163$ & $177-248$ \\
$10^{-6} \mathrm{~Hz}-10^{-1} \mathrm{~Hz} ; \rightarrow \mathrm{II}$ & $43-64$ & $70-104$ & $118-173$ & $192-280$ \\
$10^{-6} \mathrm{~Hz}-10^{-2} \mathrm{~Hz}-10^{-1} \mathrm{~Hz} ; \rightarrow \mathrm{III}$ & $37-55$ & $61-90$ & $104-150$ & $171-242$ \\
$10^{-6} \mathrm{~Hz}-10^{-4} \mathrm{~Hz}-10^{-2} \mathrm{~Hz} ; \rightarrow \mathrm{IV}$ & $34-50$ & $54-79$ & $86-125$ & $131-186$ \\
$10^{-6} \mathrm{~Hz}-10^{-2} \mathrm{~Hz}-10^{-1} \mathrm{~Hz}, L(r) ; \rightarrow \mathrm{V}$ & $38-56$ & $63-92$ & $110-160$ & $191-276$ \\
$10^{-6} \mathrm{~Hz}-10^{-4} \mathrm{~Hz}-10^{-2} \mathrm{~Hz}, L(r) ; \rightarrow \mathrm{VI}$ & $35-51$ & $56-82$ & $92-133$ & $146-208$ \\
\hline $\mathrm{I}$ and II, Power-law & $54-80$ & $85-126$ & $130-191$ & $187-272$ \\
III and IV, Power-law & $54-80$ & $85-126$ & $131-191$ & $187-271$ \\
$\mathrm{~V}$ and VI, Power-law & $56-82$ & $89-129$ & $139-200$ & $204-289$ \\
\hline
\end{tabular}

